\title{
Image Estimation from Marker Locations for Dose Calculation in Prostate Radiation Therapy
}

\author{
Huai-Ping Lee ${ }^{1}$, Mark Foskey ${ }^{1}$, Josh Levy $^{3}$, Rohit Saboo ${ }^{1}$, and Ed Chaney ${ }^{2,3}$ \\ ${ }^{1}$ Dept. of Computer Science, \\ ${ }^{2}$ Dept. of Radiation Oncology, University of North Carolina at Chapel Hill, USA \\ ${ }^{3}$ Morphormics Inc, Chapel Hill, USA
}

\begin{abstract}
Tracking implanted markers in the prostate during each radiation treatment delivery provides an accurate approximation of prostate location, which enables the use of higher daily doses with tighter margins of the treatment beams and thus improves the efficiency of the radiotherapy. However, the lack of 3D image data with such a technique prevents calculation of delivered dose as required for adaptive planning. We propose to use a reference statistical shape model generated from the planning image and a deformed version of the reference model fitted to the implanted marker locations during treatment to estimate a regionally dense deformation from the planning space to the treatment space. Our method provides a means of estimating the treatment image by mapping planning image data to treatment space via the deformation field and therefore enables the calculation of dose distributions with marker tracking techniques during each treatment delivery.
\end{abstract}

\section{Introduction}

In order to deliver a high dose of radiation to cancerous tissue while sparing nearby normal tissues, modern radiotherapy protocols create steep dose gradients near the boundary of the target volume (we consider the prostate in this paper). These techniques, however, are very sensitive to treatment uncertainties such as day-to-day changes in the geometry of the internal organs, because they have only a narrow margin of high dose around the target volume. To cope with this difficulty, image-guided radiotherapy (IGRT) [1 uses a CT image taken at planning time (the planning image) and a CT image taken immediately before a dose fraction (a treatment image) to detect potential positioning errors and changes in anatomic geometry relative to the planning image. Patients are realigned and beam apertures are reshaped to correct for these errors prior to treatment. Treatment images can also be used to calculate and assess the delivered dose in the context of adaptive radiation therapy, a form of treatment that compensates for differences between planned and delivered doses. The delivered dose is calculated in the treatment space given the known treatment parameters. A non-rigid transformation from the treatment space to the planning space is established by mapping the treatment image to the planning image using an image registration method [23]. The transformation for each treatment day is

T. Jiang et al. (Eds.): MICCAI 2010, Part III, LNCS 6363, pp. 335-342, 2010.

(C) Springer-Verlag Berlin Heidelberg 2010 
then used to deform the radiation dose calculated for each treatment day to the planning space, where it can be added to dose delivered on other days. The result can then be used if necessary to modify the treatment plan to compensate for any discrepancy between the planned and actual cumulative delivered doses [4. A problem with this approach is that the patient geometry is not sampled during actual treatment delivery [5]6].

An alternative method to monitor the prostate motion is by tracking several (usually three) markers that are implanted in the prostate. For example, in the Calypso system, the markers take the form of electromagnetic transponders that can be tracked to sub-millimeter accuracy at a sample rate of $10 \mathrm{~Hz}$, using a GPS-like system 78. The locations of the markers are used to position the patient accurately in the treatment machine, thus eliminating the need for image guidance or external marks on the patient's skin or fixation device. Although the tracking information is accurate and takes intra-treatment motion into account, the image data needed for dose calculation is missing, eliminating the promise of calculating and accumulating delivered dose. In this paper, we demonstrate that the non-rigid mapping from the planning space to the treatment space can be inferred in a neighborhood of the prostate from the three marker locations and used to estimate the missing treatment image data from the planning image.

Our problem is different from the image registration problem in that the image data on the treatment day is missing, therefore the methods based on voxelscale intensity matching [3] or based on surface matching [9] cannot be applied. Landmark-based registration methods [10] require much more than three marker locations to interpolate the deformation field and are not directly applicable to our problem. Our approach is to use a statistical shape model for the prostate so that the most likely prostate shape can be estimated given the measured marker locations. As our shape model we choose to use the $m$-rep (medial representation) [11 because it provides a coordinate system that can represent the interior and the nearby exterior of the object, so that the markers in the prostate can be also represented relative to an m-rep model of the prostate.

We create a reference m-rep from the planning image and one for each treatment day, based on the marker locations. The deformation between the reference m-rep and estimated treatment m-rep provides a transformation between the planning space and the treatment space that can be used to estimate the treatment image to calculate delivered dose. We compare the estimated images with the real treatment images to demonstrate the ability to reconstruct treatment images, and we compare the dose histograms computed using the estimated treatment images to the histograms generated by an IGRT procedure (using actual CT images) to establish the feasibility of dose calculation using estimated treatment images.

\section{Method}

Given the planning image, a reference m-rep of the prostate is created based on the gray-scale image and several user-specified landmarks on the organ 
boundary [12. Calypso markers are $\sim 8.5 \mathrm{~mm}$ in length and $\sim 1.5 \mathrm{~mm}$ in diameter that appear as bright spots in the image data. The location of each marker is computed as the center of mass of the bright spot, which closely corresponds to the origin of signals emitted by the miniature electromagnetic coil housed in the marker. For each treatment fraction, the three marker locations are given by the Calypso system, and the reference m-rep is then fitted to those locations by an optimization procedure. This procedure is similar to organ segmentation except that the seed locations, rather than image intensities, are what drive deformation within the trained shape space describing day-to-day shape variations of the prostate 12. The diffeomorphism implied by the two m-reps is then computed with a shape interpolation method called rotational flows [13. The deformation is used to transform the planning image to generate an estimated treatment image for dose calculation.

\subsection{M-reps}

The discrete m-rep [11] can be thought of as a discrete generalization of the medial axis [14. A simple m-rep model consists of a discretely sampled medial surface. Sample points are referred to as hubs, and associated to each hub are two vectors (known as spokes) extending, on opposite sides of the medial sheet, from the hub to the boundary of the modeled object. The model is trained from a population of shapes by adjusting the parameters (the locations of the hubs and the directions and lengths of the spokes) to make the model fit each shape. From this data, a mean shape and its modes of variation can be determined [15]. This statistical representation can be used to reduce the effective dimension of the shape space, so that the limited information available from the three marker locations can be applied most effectively. For instance, changes orthogonal to the plane of the three points may be implied by their relative motions, given the statistical behavior of the shape space, even though that orthogonal direction is not explicitly sampled. At planning time, a patient-specific m-rep is created from the planning image for each patient. For each treatment fraction, the patientspecific m-rep is deformed using the modes of variation to fit the treatment image (in an IGRT) or the marker locations measured for each treatment fraction (in the case of using markers instead of treatment images).

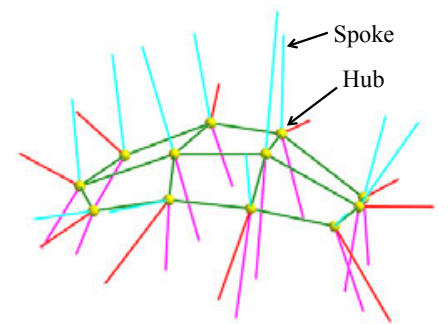

Fig. 1. A simple $3 \times 4 \mathrm{~m}$-rep. A single shape is represented by the locations of the hubs and the lengths and directions of the spokes. The boundary of the object (not shown) passes through the ends of the spokes. 
An object-relative coordinate representation (figural coordinates) of the interior and nearby exterior of the organ is used to interpolate the locations of unsampled spokes. Two coordinates, $u$ and $v$, reflect the arrangement of the hubs on the medial surface. Each hub, along with its associated spokes determines integer values for $u$ and $v$, and points between them have non-integer values. An additional coordinate, $\phi$, is equal to 1 along the entire top surface (bounded by the upper spokes extending from the outermost hubs) and -1 along the bottom surface, varying smoothly between those values over the intermediate portion of the boundary. A fourth coordinate, $\tau$, gives the fraction of the distance from the medial sheet to the boundary of the organ. This fraction may be greater than 1 for points outside the organ.

\subsection{Rotational Flows and Deformation Field Generation}

Given the reference m-rep $m_{0}$ and the target m-rep $m_{1}$ created by fitting $m_{0}$ to the marker locations, the deformation from $m_{0}$ into $m_{1}$ is computed by interpolating between the sets of boundary points $b_{0}$ and $b_{1}$, which are sampled using the same set of figural coordinates $\left\{\left(u_{i}, v_{i}, \phi_{i}\right) \mid i=1, \ldots, n\right\}$, on the boundary of $m_{0}$ and $m_{1}$, respectively. Thus for each point $\mathbf{x}_{0}$ in the sets $b_{0}$, there is a point $\mathbf{x}_{1}$ in $b_{1}$ that has the correspondence with $\mathbf{x}_{0}$ given by the m-reps and the figural coordinates. The goal of the rotational flows method is to interpolate between each pair of corresponding oriented vertices in $\mathbb{R}^{3},\left(\mathbf{x}_{0}, \mathbf{E}_{0}\right)$ and $\left(\mathbf{x}_{1}, \mathbf{E}_{1}\right)$, where $\mathbf{E}_{i}=\left\{\mathbf{e}_{1}, \mathbf{e}_{2}, \mathbf{e}_{3}\right\}$ is the set of orthonormal bases representing the orientation for the point $\mathbf{x}_{i}$. In $3 \mathrm{D}$ cases, each oriented vertex moves along a helical path along the axis of rotation. It can be shown that the interpolation is shape-maintaining if the two objects are similar and is size-maintaining if the two models are congruent [13.

Once the curved path for each pair of corresponding points is computed, the deformation for each voxel is computed by numerically integrating the subdivided displacement along each curved path. At each integration step, a radial basis function (RBF) interpolation is used to compute the deformation field in a rectangular box containing the prostate with a small margin, using the collection of landmarks. Some static landmarks are added on the boundary of the box to make the transition across the box boundary smoother. The deformation outside of the rectangular box is assumed to be zero. Due to the physics of dose deposition at high energies, this assumption has negligible effect on the accuracy of dose calculation.

\subsection{Dose Calculation}

Calculation of delivered dose is a well-established part of radiation therapy planning. CT intensities are based on the absorptivity of tissue to radiation, so it is possible to calculate the amount of energy a given beam deposits in each portion of tissue it passes through or near, taking into account the attenuation of the beam and the scattering of radiation into neighboring tissue. We use the treatment planning system known as PlanUNC [16] to calculate dose. 
Dose calculations for treatment planning are expected to be $\pm 3 \%$ compared to measurements.

These calculations are most commonly used in treatment planning. But when images are available for treatment fractions, dose can be calculated for those days too. Then, if a mapping can be constructed between the image of each treatment fraction and a common reference frame, the dose can be accumulated to determine the total amount of radiation received by each portion of the tissue. Our approach is a reversal of the most common methods of dose accumulation. Taking the planning image as given, we derive a deformation first and derive the treatment image from it, rather than acquiring a treatment image first and then deriving the deformation.

\section{Experiments}

We evaluated our method on four patient data sets, which contain one, eight, six, and five treatment fractions, respectively. The data for each patient consist of a planning image, treatment images, and Calypso marker locations during treatment. Note that for each treatment fraction, the patient is positioned in the treatment machine according to the Calypso origin, which also serves as the isocenter of the treatment plan (i.e., the point in the space where the central beam of radiation passes), and treatment images are taken only for comparison with estimated images. Since the treatment images are taken before the patient is positioned for treatment, they are visually aligned to the Calypso marker locations before the comparison with estimated images and the non-rigid image registration for dose calculation.

In order to assess the similarity between the estimated image and real treatment image, we create an m-rep of the prostate for each treatment image based on the gray-scale image and user-specified landmarks. The estimated m-rep ( $m_{\text {marker }}$, reference m-rep fitted to the marker locations) is then compared to the treatment m-rep $\left(m_{\text {treat }}\right.$, m-rep created with the treatment image) by computing the distance between sample points on the surfaces. For each sample point $p$ on the surface of $m_{\text {treat }}$, its distance to the m-rep $m_{\text {marker }}$ is approximated as $D\left(p, B_{\text {marker }}\right)=\min \left\{d(p, q) \mid q \in B_{\text {marker }}\right\}$, where $B_{\text {marker }}$ is the set of sample points on the surface of $m_{\text {marker }}$, and $d(p, q)$ is the Euclidean distance between the two points $p$ and $q$. The average distances from the estimated m-rep to the treatment m-rep are $0.15 \mathrm{~cm}, 0.12 \mathrm{~cm}, 0.13 \mathrm{~cm}$, and $0.10 \mathrm{~cm}$ for the four patient data sets, respectively. Note that these average distances are within the image resolution of the four data sets; two of them are $0.12 \times 0.12 \times 0.3 \mathrm{~cm}$, and the other two are $0.12 \times 0.12 \times 0.3 \mathrm{~cm}$. Fig. 2 shows an example visual comparison of the estimated image and corresponding treatment image.

The dose calculation using the estimated image is compared against the dose calculated with real treatment images to evaluate the accuracy of our method. The non-rigid transformation with the treatment images is computed in the same manner as with the estimated image, as described in Section 2.2. For the first patient data set, we consider the dose in the prostate. For the other data 


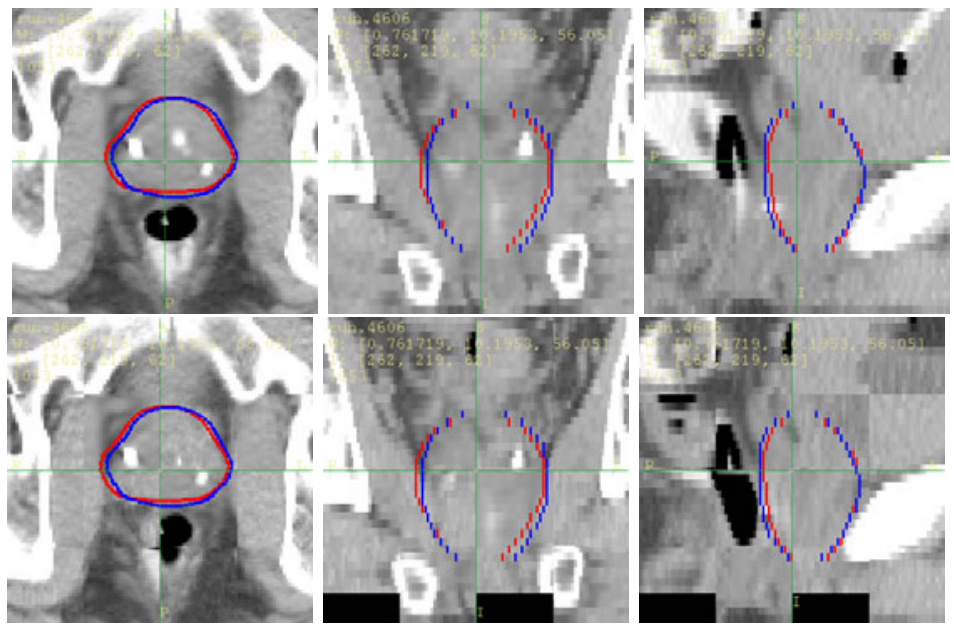

Fig. 2. Axial (left), coronal (center), and sagittal (right) views of an example mrep comparison; blue contour: m-rep fitted to Calypso marker locations during the treatment fraction; red contour: m-rep created with real treatment image; the green crosshairs show the Calypso origin, which also serves as the isocenter of the treatment plan; the CT image shown are the estimated image (top row) and the $4 \times 4$ checkerboard image comparing the estimated image to the real treatment image (bottom row).

sets, we consider the dose in the prostate and in the anterior rectal wall (the part of the rectum right next to the prostate). Fig. 3 shows the differential and cumulative dose-volume histograms (DVH) of one of the data sets using the estimated images and real treatment images. Besides visual similarity, we also numerically compare the DVHs by considering the equivalent uniform dose (EUD) 17] of the differential DVHs, as shown in Table1, along with the errors of the values given by our method relative to those given by the real images. There are no generally accepted standards for errors in calculating delivered dose, but $\pm 5 \%$ is a reasonable goal. We observed relative errors of less than $3 \%$ in EUD for the four patient data sets we experimented on.

Table 1. Comparison of equivalent uniform dose (normalized to percentage of reference dose) of the dose-volume histograms for the four patient data sets, and the errors relative to the values given by the IGRT

\begin{tabular}{|c|c|c|c||c|c|c|}
\hline \multirow{2}{*}{ Patient } & \multicolumn{3}{|c||}{ Prostate EUD } & \multicolumn{3}{c|}{ Anterior Rectal Wall EUD } \\
\cline { 2 - 7 } & Tx Image & Est. Image & Error & Tx Image & Est. Image & Error \\
\hline \hline 1 & 96.93 & 98.17 & $1.28 \%$ & N/A & N/A & N/A \\
\hline 2 & 102.54 & 102.51 & $-0.02 \%$ & 102.04 & 101.55 & $-0.49 \%$ \\
\hline 3 & 98.68 & 100.71 & $2.06 \%$ & 92.42 & 92.20 & $-0.24 \%$ \\
\hline 4 & 102.62 & 102.59 & $-0.03 \%$ & 101.72 & 101.21 & $-0.50 \%$ \\
\hline
\end{tabular}



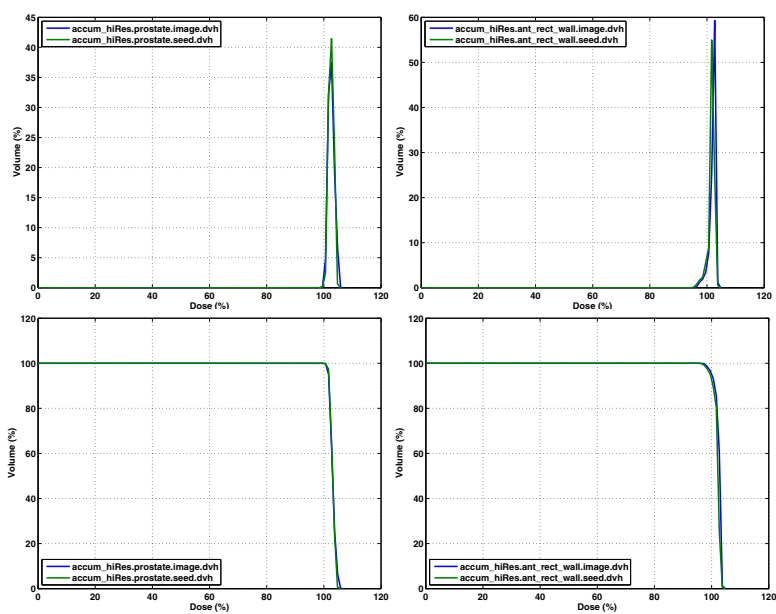

Fig. 3. Differential (top row) and cumulative (bottom row) dose-volume histograms for the prostate (left) and anterior rectal wall (right); blue lines represent results with real treatment images, and green lines represents results with estimated images

\section{Conclusion}

We presented a method to estimate the treatment image using the planning image and locations of implanted markers during the treatment. We demonstrated the feasibility of image estimation by comparing the estimated images to real treatment images, and we also showed that the calculated dose histograms using the estimated images are close to those using real treatment images. Our method complements the Calypso system, where the prostate motion can be tracked accurately at a high frequency during the treatment but the image data is missing, so that the delivered dose distribution can be calculated and a safe delivery can be insured.

In the future, we will experiment on more patient data and assess different deformation methods such as FEM-based methods. We will also investigate the feasibility of adjusting the treatment machine to compensate for the intratreatment motion observed.

Acknowledgements. This work was supported by funds from grant 1R43CA141941 to Morphormics, Inc. from the US National Cancer Institute, and by a research agreement with Calypso Medical.

\section{References}

1. Wong, J.R., Grimm, L., Uematsu, M., Oren, R., Cheng, C.W., Merrick, S., Schiff, P.: Image-guided radiotherapy for prostate cancer by CT-linear accelerator combination: Prostate movements and dosimetric considerations. International Journal of Radiation Oncology*Biology*Physics 61(2), 561-569 (2005) 
2. Maintz, J., Viergever, M.A.: A survey of medical image registration. Medical Image Analysis 2(1), 1-36 (1998)

3. Foskey, M., Davis, B., Goyal, L., Chang, S., Chaney, E., Strehl, N., Tomei, S., Rosenman, J., Joshi, S.: Large deformation Three-Dimensional image registration in Image-Guided radiation therapy. Physics in Medicine and Biology 50, 5869-5892 (2005)

4. Yan, D., Lockman, D., Brabbins, D., Tyburski, L., Martinez, A.: An off-line strategy for constructing a patient-specific planning target volume in adaptive treatment process for prostate cancer. International Journal of Radiation Oncology*Biology*Physics 48(1), 289-302 (2000)

5. Litzenberg, D.W., Hadley, S.W., Tyagi, N., Balter, J.M., Haken, R.K.T., Chetty, I.J.: Synchronized dynamic dose reconstruction. Medical Physics 34(1), 91-102 (2007)

6. Noel, C., Parikh, P.J., Roy, M., Kupelian, P., Mahadevan, A., Weinstein, G., Enke, C., Flores, N., Beyer, D., Levine, L.: Prediction of intrafraction prostate motion: Accuracy of pre- and Post-Treatment imaging and intermittent imaging. International Journal of Radiation Oncology Biology Physics 73(3), 692-698 (2009)

7. Langen, K.M., Willoughby, T.R., Meeks, S.L., Santhanam, A., Cunningham, A., Levine, L., Kupelian, P.A.: Observations on real-time prostate gland motion using electromagnetic tracking. International Journal of Radiation Oncology, Biology, Physics 71(4), 1084-1090 (2008); PMID: 18280057

8. Balter, J.M., Wright, J.N., Newell, L.J., Friemel, B., Dimmer, S., Cheng, Y., Wong, J., Vertatschitsch, E., Mate, T.P.: Accuracy of a wireless localization system for radiotherapy. International Journal of Radiation Oncology, Biology, Physics 61(3), 933-937 (2005); PMID: 15708277

9. Kaus, M.R., Brock, K.K., Pekar, V., Dawson, L.A., Nichol, A.M., Jaffray, D.A.: Assessment of a Model-Based deformable image registration approach for radiation therapy planning. Int. J. Radiation Oncology Bio. Phys. 68(2), 572-580 (2007)

10. Rohr, K., Stiehl, H.S., Sprengel, R., Buzug, T.M., Weese, J., Kuhn, M.H.: Landmark-based elastic registration using approximating thin-plate splines. IEEE Transactions on Medical Imaging 20(6), 526-534 (2001)

11. Pizer, S.M., Fletcher, P.T., Joshi, S., Thall, A., Chen, J.Z., Fridman, Y., Fritsch, D.S., Gash, A.G., Glotzer, J.M., Jiroutek, M.R., Lu, C., Muller, K.E., Tracton, G., Yushkevich, P., Chaney, E.L.: Deformable M-Reps for 3D medical image segmentation. Int. J. Comput. Vision 55(2-3), 85-106 (2003)

12. Pizer, S.M., Fletcher, P.T., Joshi, S., Gash, A.G., Stough, J., Thall, A., Tracton, G., Chaney, E.L.: A method and software for segmentation of anatomic object ensembles by deformable m-reps. Medical Physics 32(5), 1335-1345 (2005)

13. Levy, J.H., Foskey, M., Pizer, S.M.: Rotational flows for interpolation between sampled surfaces. In: Computer Vision and Pattern Recognition Workshop, pp. 1-8. IEEE Computer Society, Los Alamitos (2008)

14. Blum, H., Wathen-Dunn, W.: A transformation for extracting new descriptors of shape. Models for the Perception of Speech and Visual Form, 362-380 (1967)

15. Merck, D., Tracton, G., Saboo, R., Levy, J., Chaney, E., Pizer, S., Joshi, S.: Training models of anatomic shape variability. Medical Physics 35(8), 3584-3596 (2008)

16. Schreiber, E., Xu, Z., Lorenzen, A., Foskey, M., Cullip, T., Tracton, G., Chaney, E.: SU-FF-T-362: PLanUNC as an Open-Source radiotherapy planning system for research and education. Medical Physics 33(6), 2129 (2006)

17. Wu, Q., Mohan, R., Niemierko, A., Schmidt-Ullrich, R.: Optimization of intensitymodulated radiotherapy plans based on the equivalent uniform dose. International Journal of Radiation Oncology, Biology, Physics 52(1), 224-235 (2002) 